\title{
Irrigation and Fertilizers Management Practices in Maize (Zea mays L.) and their Residual Effect on Harvest Index and Soil Moisture Studies of Soybean (Glycine max L.)
}

\author{
Sangh Ravikiran* and S. S. Angadi \\ Department of Agronomy, College of Agriculture, University of Agricultural Sciences, \\ Dharwad-580 005, Karnataka, India \\ *Corresponding author
}

\section{A B S T R A C T}

Keywords

Fertilizer, Hydrogel, Irrigation, Residual effect and Time of application

Article Info

Accepted:

22 January 2021

Available Online:

10 February 2021
Experiment was conducted in sequence cropping (Maize - Soybean) with Soybean as residual crop in Kharif at Dharwad on clayey texture in Split split plot with three replications during 2013-14 and 2014-15. Harvest index did not differed significantly with residual effect of irrigation levels. Whereas, residual effect due to fertilizers levels of 250:100:125 kg $\mathrm{N}: \mathrm{P}_{2} \mathrm{O}_{5}: \mathrm{K}_{2} \mathrm{O}$ ha $^{-1}(2.5: 1: 1.25)$ recorded significantly higher harvest index (0.41). Similarly, residual effect of fertilizers application as $33 \%$ each $\left(1 / 3^{\text {rd }}\right)$ of $\mathrm{N}, \mathrm{P}_{2} \mathrm{O}_{5}$ and $\mathrm{K}_{2} \mathrm{O}$ as basal, $33 \%$ each of $\mathrm{N}, \mathrm{P}_{2} \mathrm{O}_{5}$ and $\mathrm{K}_{2} \mathrm{O}$ at 30 DAS and $33 \%$ each of $\mathrm{N}, \mathrm{P}_{2} \mathrm{O}_{5}$ and $\mathrm{K}_{2} \mathrm{O}$ at 50 DAS recorded higher harvest index (0.40). Besides, residual effect of interaction due to irrigation and fertilizer management practices did not differed significantly on harvest index.

\section{Introduction}

From last few years, nutrient research has been shifted from individual crop to cropping system. Presently, efficiency of nutrients (N $50 \%, \mathrm{P}_{2} \mathrm{O}_{5}-25 \%$ and $\mathrm{K}_{2} \mathrm{O}-30 \%$ ) is usually less and remaining nutrients are left over in the soil unless managed properly. Water (reduced water application strategies) and nutrient management practices (adequate and balanced fertilization with split application of fertilizers), rainfall intensity, soil type, root system and genotype efficiency are the factor which decides availability of cumulative nutrients in the soil. Moreover, water is a scarce input and its management places an important for increasing efficiency of water and nutrients. Adoption of conservation tillage practices reduces decomposition of organic matter and conserves water and nutrients in the soil, besides sustaining short and long term needs 
of soil and crop. Hence short duration pulses which require less fertilizer and water can be grown on left over soil moisture and nutrients for economization of resources. Therefore, the present study was planned to examine the above said problems for increasing the productivity.

\section{Materials and Methods}

Residual research was carried out in Kharif Soybean at Main Agricultural Research Station, Dharwad on clayey texture. Before experimentation, soil sampling of the plot recorded $\mathrm{pH}(7.4)$, EC $(0.23 \mathrm{dS} / \mathrm{m})$, organic carbon (0.55), available $\mathrm{N}\left(250 \mathrm{~kg} \mathrm{ha}^{-1}\right)$, available $\mathrm{P}_{2} \mathrm{O}_{5}\left(33 \mathrm{~kg} \mathrm{ha}^{-1}\right)$, available $\mathrm{K}_{2} \mathrm{O}$ $\left(290 \mathrm{~kg} \mathrm{ha}^{-1}\right)$, exchangeable $\mathrm{Ca}(28 \mathrm{cmol}(\mathrm{p}+)$ $\left.\mathrm{kg}^{-1}\right)$, exchangeable $\mathrm{Mg}\left(7 \mathrm{cmol}(\mathrm{p}+) \mathrm{kg}^{-1}\right)$ and available $\mathrm{S}\left(21 \mathrm{~kg} \mathrm{ha}^{-1}\right)$. Experiment was laid out in split split plot design with three replications. Details of treatment are presented in Table 1. Pre-plant (13 $\mathrm{ml}$ of glyphosate per litre of water), pre-emergence $(4 \mathrm{ml}$ of alachlor per litre of water) and post-emergence herbicides $(1 \mathrm{ml}$ of imazethapyr per litre of water) were used for managing weeds. Seeds (JS 335) inoculated with Rhizobium (500 $\mathrm{g} \mathrm{ha}^{-}$ $\left.{ }^{1}\right)$ were sown with interow of $30 \mathrm{~cm}$ and intrarow of $10 \mathrm{~cm}$. Harvest index was calculated by dividing yield of seed by haulm (Donald, 1962). Besides, soil moisture was estimated by Theta probe from 0-30 and 30-60 $\mathrm{cm}$ soil depth. Data were analyzed as per Gomez and Gomez (1984).

\section{Results and Discussion}

\section{Harvest index}

Irrigation and fertilizer management practices in Maize differed significantly on residual Soybean (Table 1). Residual effect of irrigation levels did not differed significantly with values ranging from 0.38 to 0.40 . Residual effect of fertilizer levels differed significantly with higher harvest index (0.41) from fertilizers dose of 250:100:125 kg $\mathrm{N}: \mathrm{P}_{2} \mathrm{O}_{5}: \mathrm{K}_{2} \mathrm{O}$ ha ${ }^{-1}$ (2.5:1:1.25). Residual effect of fertilizer applications differed significantly with higher harvest index (0.40) with application of fertilizers as $33 \%$ each $\left(1 / 3^{\text {rd }}\right)$ of $\mathrm{N}, \mathrm{P}_{2} \mathrm{O}_{5}$ and $\mathrm{K}_{2} \mathrm{O}$ as basal, $33 \%$ each of $\mathrm{N}$, $\mathrm{P}_{2} \mathrm{O}_{5}$ and $\mathrm{K}_{2} \mathrm{O}$ at 30 DAS and $33 \%$ each of $\mathrm{N}$, $\mathrm{P}_{2} \mathrm{O}_{5}$ and $\mathrm{K}_{2} \mathrm{O}$ at 50 DAS. Besides, residual interaction effect of irrigattion and fertilizer management practices did not differed significantly with values ranging from 0.36 to 0.43 . Besides, residual interaction of deficit irrigation at growth stages and fertilizer levels of 250:100:125 kg N: $\mathrm{P}_{2} \mathrm{O}_{5}: \mathrm{K}_{2} \mathrm{O} \quad \mathrm{ha}^{-1}$ $(2.5: 1: 1.25)$ when applied as $33 \%$ each $\left(1 / 3^{\text {rd }}\right)$ of $\mathrm{N}, \mathrm{P}_{2} \mathrm{O}_{5}$ and $\mathrm{K}_{2} \mathrm{O}$ as basal, $33 \%$ each of $\mathrm{N}$, $\mathrm{P}_{2} \mathrm{O}_{5}$ and $\mathrm{K}_{2} \mathrm{O}$ at 30 DAS and $33 \%$ each of $\mathrm{N}$, $\mathrm{P}_{2} \mathrm{O}_{5}$ and $\mathrm{K}_{2} \mathrm{O}$ at 50 DAS recorded higher harvest index (0.43). Higher harvest index might be due to significant dry matter accumulation in vegetative and reproductive parts which resulted in superior seed and haulm yield under suitable climatic conditions. Increased yield is due to higher residual moisture and nutrient resources as a result of higher depth of irrigation and fertilizer management practices in Maize. Similar results were recorded by De (2003), Singh et al., (2009) and Bhattacharyya et al., (2010). In addition, residual effect of control recorded significantly lower harvest index $(0.26)$ due to lower residual growth resources from preceding Maize when compared to other treatment combinations.

\section{Soil moisture content at sowing}

Moisture content of soil from 0-30 and 30-60 $\mathrm{cm}$ soil depth differed significantly from the residual plot of irrigation and fertilizer management practices (Table 1). Before sowing of Soybean, from $0-30 \mathrm{~cm}$ soil depth, residual plot of irrigation levels did not differ significantly, whereas, residual plot of fertilizer levels i.e., 250:100:125 kg 
$\mathrm{N}: \mathrm{P}_{2} \mathrm{O}_{5}: \mathrm{K}_{2} \mathrm{O} \quad \mathrm{ha}^{-1} \quad(2.5: 1: 1.25) \quad$ recorded significantly higher soil moisture content (26.8\%). Besides, residual plot of fertilizer applied as $33 \%$ each $\left(1 / 3^{\text {rd }}\right)$ of $\mathrm{N}, \mathrm{P}_{2} \mathrm{O}_{5}$ and $\mathrm{K}_{2} \mathrm{O}$ as basal, 33\% each of $\mathrm{N}, \mathrm{P}_{2} \mathrm{O}_{5}$ and $\mathrm{K}_{2} \mathrm{O}$ at 30 DAS and $33 \%$ each of $\mathrm{N}, \mathrm{P}_{2} \mathrm{O}_{5}$ and $\mathrm{K}_{2} \mathrm{O}$ at 50 DAS recorded higher moisture content in the soil $(26.8 \%)$. Further, residual plot of irrigation at growth stages and fertilizer levels of 250:100:125 kg N: $\mathrm{P}_{2} \mathrm{O}_{5}: \mathrm{K}_{2} \mathrm{O} \quad \mathrm{ha}^{-1}$ (2.5:1:1.25) with applications as $33 \%$ each $\left(1 / 3^{\text {rd }}\right)$ of $\mathrm{N}, \mathrm{P}_{2} \mathrm{O}_{5}$ and $\mathrm{K}_{2} \mathrm{O}$ as basal, $33 \%$ each of $\mathrm{N}, \mathrm{P}_{2} \mathrm{O}_{5}$ and $\mathrm{K}_{2} \mathrm{O}$ at 30 DAS and $33 \%$ each of $\mathrm{N}, \mathrm{P}_{2} \mathrm{O}_{5}$ and $\mathrm{K}_{2} \mathrm{O}$ at 50 DAS recorded significantly higher soil moisture content (27.5\%) when compared to other treatment combinations.

Similar trend of results were recorded from 30 to $60 \mathrm{~cm}$ soil depth with higher soil moisture content when compared to upper layers. Higher soil moisture content might be due to Minimum tillage in Maize, soil surface covered with maize residues and reduced weed density due to pre plant and pre emergence application of herbicides.

Similar findings were recorded by Gan et al., (2016) and Shrestha and Roy (2016). In addition, residual effect of control recorded soil moisture content of 26.8 and $27.9 \%$ from 0-30 and 30-60 cm soil depth, respectively due to conventional agriculture practices.

\section{Soil Moisture content at Harvest}

Soil moisture content from $0-30$ and $30-60 \mathrm{~cm}$ soil depth differed significantly after harvesting Soybean from residual plot of irrigation and fertilizer management practices (Table 1). Soil moisture content from $0-30 \mathrm{~cm}$ soil depth after harvesting Soybean did not differ from residual plot of irrigation levels. Whereas, soil moisture content was significantly higher (19.9\%) from residual plot of fertilizer plot of 200:75:75 kg N: $\mathrm{P}_{2} \mathrm{O}_{5}: \mathrm{K}_{2} \mathrm{O}$ $\mathrm{ha}^{-1}$ (2.67:1:1). Besides residual plot of fertilizer application as $25 \%\left(1 / 4^{\text {th }}\right)$ of $\mathrm{N}, \mathrm{K}_{2} \mathrm{O}$ and $50 \%$ of $\mathrm{P}_{2} \mathrm{O}_{5}$ as basal, $50 \%$ of $\mathrm{N}, \mathrm{P}_{2} \mathrm{O}_{5}$ and $\mathrm{K}_{2} \mathrm{O}$ at 30 days after sowing and $25 \%$ of $\mathrm{N}$ and $\mathrm{K}_{2} \mathrm{O}$ at 50 days after sowing recorded significantly higher soil moisture content $(19.5 \%)$.

Low soil fertility might be the reason for low moisture efficiency by Soybean which further conserved in the soil. Residual plot of irrigation at growth stages with Hydrogel and fertilizer levels of 200:75:75 kg N: $\mathrm{P}_{2} \mathrm{O}_{5}: \mathrm{K}_{2} \mathrm{O}$ $\mathrm{ha}^{-1}$ (2.67:1:1) with fertilizer application as $25 \%\left(1 / 4^{\text {th }}\right)$ of $\mathrm{N}, \mathrm{K}_{2} \mathrm{O}$ and $50 \%$ of $\mathrm{P}_{2} \mathrm{O}_{5}$ as basal, $50 \%$ of $\mathrm{N}, \mathrm{P}_{2} \mathrm{O}_{5}$ and $\mathrm{K}_{2} \mathrm{O}$ at 30 days after sowing and $25 \%$ of $\mathrm{N}$ and $\mathrm{K}_{2} \mathrm{O}$ at 50 days after sowing documented higher soil moisture content $(20.2 \%)$ when compared to other treatment combinations. Similar pattern of results were recorded from $30-60 \mathrm{~cm}$ soil depth, but moisture content were lower when compared to upper layers.

Increased soil moisture might be due to Soybean sown under Zero tillage, partially decomposed maize residues and pre emergence and post emergence application of herbicides which reduced uptake of moisture by weeds. Similar results were documented by Ali et al., (2000), Bandyopadhyay et al., (2016), Kumar et al., (2018), and Hazra and Bohra (2021). In addition, residual effect of control recorded soil moisture content of 18.1 and $19.4 \%$ from $0-30$ and $30-60 \mathrm{~cm}$ soil depth, respectively due to conventional agriculture practices.

Residual interaction of irrigation at growth stages and fertilizer levels of 250:100:125 kg $\mathrm{N}: \mathrm{P}_{2} \mathrm{O}_{5}: \mathrm{K}_{2} \mathrm{O}$ ha $^{-1}$ (2.5:1:1.25) with fertilizers application as $33 \%$ each $\left(1 / 3^{\text {rd }}\right)$ of $\mathrm{N}, \mathrm{P}_{2} \mathrm{O}_{5}$ and $\mathrm{K}_{2} \mathrm{O}$ as basal, $33 \%$ each of $\mathrm{N}, \mathrm{P}_{2} \mathrm{O}_{5}$ and $\mathrm{K}_{2} \mathrm{O}$ at 30 DAS and $33 \%$ each of $\mathrm{N}, \mathrm{P}_{2} \mathrm{O}_{5}$ and $\mathrm{K}_{2} \mathrm{O}$ at 50 DAS performed better when compared to other treatment combinations. 
Table.1 Harvest index and Soil moisture studies of soybean (Glycine max L.) as influenced by residual moisture and nutrient resources

\begin{tabular}{|c|c|c|c|c|c|c|c|c|c|c|c|c|c|c|c|c|}
\hline & & \multirow{2}{*}{\multicolumn{3}{|c|}{ Harvest index }} & \multicolumn{6}{|c|}{ Soil moisture content at sowing } & \multicolumn{6}{|c|}{ Soil moisture content at harvest } \\
\hline & & & & & \multicolumn{3}{|c|}{ Soil moisture content at 0-30 cm } & \multicolumn{3}{|c|}{ Soil moisture content at $30-60 \mathrm{~cm}$} & \multicolumn{3}{|c|}{ Soil moisture content at $0-30 \mathrm{~cm}$} & \multicolumn{3}{|c|}{ Soil moisture content at $30-60 \mathrm{~cm}$} \\
\hline & & $\mathbf{T}_{1}$ & $\mathbf{T}_{2}$ & \multirow[t]{2}{*}{$\mathbf{I} \times \mathbf{F}$} & $\mathbf{T}_{1}$ & $\mathbf{T}_{2}$ & $\mathbf{I} \times \mathbf{F}$ & $\mathbf{T}_{1}$ & $\mathbf{T}_{2}$ & \multirow[t]{2}{*}{$\mathbf{I} \times \mathbf{F}$} & $\mathbf{T}_{1}$ & $\mathbf{T}_{2}$ & \multirow[t]{2}{*}{$\mathbf{I} \times \mathbf{F}$} & $\mathbf{T}_{1}$ & $\mathbf{T}_{2}$ & \multirow[t]{2}{*}{$\mathbf{I} \times \mathbf{F}$} \\
\hline & & & & & $\mathbf{I x}$ & & & & & & Ix & & & Ix & & \\
\hline \multirow[t]{3}{*}{$\mathbf{I}_{1}$} & $F_{1}$ & $0.36 \mathrm{a}$ & $0.39 \mathrm{a}$ & $0.38 \mathrm{ab}$ & $26.3 \mathrm{c}-\mathrm{e}$ & $26.4 \mathrm{~b}-\mathrm{e}$ & $26.3 \mathrm{~b}-\mathrm{d}$ & $27.3 \mathrm{c}-\mathrm{f}$ & $27.5 \mathrm{c}-\mathrm{f}$ & $27.4 \mathrm{bc}$ & $20.1 \mathrm{ab}$ & $19.8 \mathrm{a}-\mathrm{c}$ & 19.9ab & $21.3 \mathrm{a}$ & $20.8 \mathrm{a}-\mathrm{c}$ & 21.0ab \\
\hline & $\mathbf{F}_{2}$ & $0.39 \mathrm{a}$ & $0.41 \mathrm{a}$ & $0.40 \mathrm{ab}$ & $26.7 \mathrm{a}-\mathrm{e}$ & $27.4 \mathrm{ab}$ & $27.1 \mathrm{a}$ & $27.7 \mathrm{~b}-\mathrm{f}$ & $28.4 \mathrm{a}-\mathrm{c}$ & 28.1ab & $19.5 \mathrm{bc}$ & $18.9 \mathrm{de}$ & $19.2 \mathrm{c}$ & $20.6 b-d$ & $19.9 \mathrm{fg}$ & $20.2 \mathrm{c}$ \\
\hline & $\mathbf{F}_{3}$ & $0.40 \mathrm{a}$ & $0.43 \mathrm{a}$ & $0.42 \mathrm{a}$ & $26.5 \mathrm{a}-\mathrm{e}$ & $27.5 \mathrm{a}$ & 27.0ab & $27.9 \mathrm{a}-\mathrm{e}$ & $28.7 \mathrm{ab}$ & $28.3 \mathrm{a}$ & 18.9de & $18.4 \mathrm{e}-\mathrm{h}$ & 18.6d & $19.9 \mathrm{fg}$ & $19.3 \mathrm{~h}$ & 19.6d \\
\hline \multirow[t]{3}{*}{$\mathbf{I}_{2}$} & $\mathbf{F}_{1}$ & $0.36 \mathrm{a}$ & $0.39 \mathrm{a}$ & $0.37 \mathrm{~b}$ & $25.9 \mathrm{de}$ & $26.4 \mathrm{~b}-\mathrm{e}$ & $26.2 \mathrm{~cd}$ & $27.2 \mathrm{~d}-\mathrm{f}$ & $27.7 b-f$ & $27.5 \mathrm{bc}$ & $20.2 \mathrm{a}$ & $20.1 \mathrm{ab}$ & $20.2 \mathrm{a}$ & $21.3 \mathrm{a}$ & 21.1ab & 21.2a \\
\hline & $\mathbf{F}_{2}$ & $0.39 \mathrm{a}$ & $0.40 \mathrm{a}$ & $0.40 \mathrm{ab}$ & 26.5a-e & $27.3 \mathrm{a}-\mathrm{c}$ & $26.9 \mathrm{a}-\mathrm{c}$ & $27.2 \mathrm{~d}-\mathrm{f}$ & $28.3 a-d$ & $27.8 \mathrm{ab}$ & $19.7 \mathrm{a}-\mathrm{c}$ & $18.7 \mathrm{e}-\mathrm{g}$ & $19.2 \mathrm{c}$ & $20.5 \mathrm{c}-\mathrm{e}$ & $19.5 \mathrm{gh}$ & $20.0 \mathrm{c}$ \\
\hline & $\mathbf{F}_{3}$ & $0.40 \mathrm{a}$ & $0.41 \mathrm{a}$ & $0.40 \mathrm{ab}$ & $26.3 \mathrm{c}-\mathrm{e}$ & $27.4 \mathrm{ab}$ & $26.9 a-c$ & $27.7 b-f$ & $28.9 \mathrm{a}$ & $28.3 \mathrm{a}$ & $19.3 \mathrm{~cd}$ & $18.1 \mathrm{~h}$ & $18.7 \mathrm{~d}$ & $20.2 \mathrm{~d}-\mathrm{f}$ & $19.1 \mathrm{~h}$ & 19.6d \\
\hline \multirow[t]{14}{*}{$\mathbf{I}_{3}$} & $\mathbf{F}_{1}$ & $0.36 \mathrm{a}$ & $0.37 \mathrm{a}$ & $0.36 \mathrm{~b}$ & $25.7 \mathrm{e}$ & $25.8 \mathrm{e}$ & $25.8 \mathrm{~d}$ & $26.6 f$ & 27.1ef & $26.8 \mathrm{c}$ & 19.9ab & $19.6 a-c$ & $19.7 \mathrm{~b}$ & $20.9 \mathrm{a}-\mathrm{c}$ & $20.5 \mathrm{c}-\mathrm{e}$ & $20.7 b$ \\
\hline & $\mathbf{F}_{2}$ & $0.38 \mathrm{a}$ & $0.40 \mathrm{a}$ & $0.39 \mathrm{ab}$ & $26.4 \mathrm{~b}-\mathrm{e}$ & 26.0de & $26.2 \mathrm{~cd}$ & $27.5 \mathrm{c}-\mathrm{f}$ & $27.5 c-f$ & $27.5 b c$ & $19.3 \mathrm{~cd}$ & $18.3 \mathrm{f}-\mathrm{h}$ & $18.8 \mathrm{~d}$ & $20.0 \mathrm{e}-\mathrm{g}$ & $19.3 \mathrm{~h}$ & 19.6d \\
\hline & $F_{3}$ & $0.39 \mathrm{a}$ & $0.42 \mathrm{a}$ & $0.40 \mathrm{ab}$ & $26.2 \mathrm{de}$ & 26.9a-d & $26.6 \mathrm{a}-\mathrm{c}$ & $27.5 \mathrm{c}-\mathrm{f}$ & 27.9a-e & $27.7 \mathrm{ab}$ & $18.8 \mathrm{~d}-\mathrm{f}$ & $18.2 \mathrm{gh}$ & $18.5 \mathrm{~d}$ & $19.6 \mathrm{gh}$ & $19.3 \mathrm{~h}$ & 19.4d \\
\hline & & $\mathbf{T}_{1}$ & $\mathbf{T}_{2}$ & \multirow[t]{2}{*}{$\mathbf{I}$} & $\mathbf{T}_{1}$ & $\mathbf{T}_{2}$ & I & $\mathbf{T}_{1}$ & $\mathbf{T}_{2}$ & \multirow[t]{2}{*}{$\mathbf{I}$} & $T_{1}$ & $\mathbf{T}_{2}$ & \multirow[t]{2}{*}{$\mathbf{I}$} & $\mathbf{T}_{1}$ & $\mathbf{T}_{2}$ & \multirow[t]{2}{*}{ I } \\
\hline & & \multicolumn{2}{|c|}{$\mathbf{I} \times \mathbf{T}$} & & \multicolumn{2}{|c|}{$\mathbf{I} \times \mathbf{T}$} & & \multicolumn{2}{|c|}{$\mathbf{I} \times \mathbf{T}$} & & \multicolumn{2}{|c|}{$\mathbf{I} \times \mathbf{T}$} & & \multicolumn{2}{|c|}{$\mathbf{I} \times \mathbf{T}$} & \\
\hline & $\mathbf{I}_{1}$ & $0.39 \mathrm{ab}$ & $0.41 \mathrm{a}$ & $0.40 \mathrm{a}$ & $26.5 b c$ & 27.1a & $26.8 \mathrm{a}$ & $27.6 b$ & $28.2 \mathrm{a}$ & $27.9 \mathrm{a}$ & $19.5 \mathrm{ab}$ & $19.0 \mathrm{~cd}$ & $19.2 \mathrm{a}$ & $20.6 \mathrm{a}$ & $20.0 \mathrm{bc}$ & 20.3a \\
\hline & $\mathbf{I}_{2}$ & $0.38 \mathrm{ab}$ & $0.40 \mathrm{ab}$ & $0.39 \mathrm{a}$ & $26.2 \mathrm{c}$ & 27.0ab & $26.6 \mathrm{a}$ & $27.4 \mathrm{~b}$ & $28.3 \mathrm{a}$ & $27.8 \mathrm{a}$ & $19.7 \mathrm{a}$ & $19.0 \mathrm{~cd}$ & $19.4 \mathrm{a}$ & $20.7 \mathrm{a}$ & $19.9 \mathrm{bc}$ & 20.3a \\
\hline & $\mathbf{I}_{3}$ & $0.37 \mathrm{~b}$ & $0.39 \mathrm{ab}$ & $0.38 \mathrm{a}$ & $26.1 \mathrm{c}$ & $26.2 \mathrm{c}$ & $26.2 \mathrm{a}$ & $27.2 b$ & $27.5 b$ & $27.3 \mathrm{a}$ & $19.3 \mathrm{bc}$ & $18.7 \mathrm{~d}$ & $19.0 \mathrm{a}$ & $20.2 b$ & $19.7 \mathrm{c}$ & 19.9a \\
\hline & & $\mathbf{T}_{1}$ & $\mathbf{T}_{2}$ & \multirow[t]{2}{*}{$\mathbf{F}$} & $\mathbf{T}_{1}$ & $\mathbf{T}_{2}$ & $\mathbf{F}$ & $\mathbf{T}_{1}$ & $\mathbf{T}_{2}$ & $\mathbf{F}$ & $\mathbf{T}_{1}$ & $\mathbf{T}_{2}$ & $\mathbf{F}$ & $\mathbf{T}_{1}$ & $\mathbf{T}_{2}$ & $\mathbf{F}$ \\
\hline & & & & & & & & & & & & & & & & \\
\hline & $\mathbf{F}_{1}$ & $0.36 c$ & $0.38 b c$ & $0.37 \mathrm{~b}$ & $26.0 \mathrm{c}$ & $26.2 \mathrm{c}$ & $26.1 \mathrm{~b}$ & $27.0 \mathrm{~d}$ & $27.4 \mathrm{~cd}$ & $27.2 b$ & $20.1 \mathrm{a}$ & 19.8ab & 19.9a & $21.1 \mathrm{a}$ & $20.8 \mathrm{a}$ & 21.0a \\
\hline & $\mathbf{F}_{2}$ & $0.39 a-c$ & $0.40 \mathrm{ab}$ & $0.39 \mathrm{ab}$ & $26.5 b c$ & $26.9 \mathrm{ab}$ & $26.7 \mathrm{a}$ & $27.5 \mathrm{~cd}$ & $28.1 \mathrm{ab}$ & $27.8 \mathrm{a}$ & $19.5 b$ & $18.6 \mathrm{~d}$ & $19.1 \mathrm{~b}$ & $20.4 b$ & $19.5 \mathrm{~d}$ & $20.0 \mathrm{~b}$ \\
\hline & $\mathbf{F}_{3}$ & $0.39 a-c$ & $0.42 \mathrm{a}$ & $0.41 \mathrm{a}$ & $26.4 \mathrm{bc}$ & $27.3 \mathrm{a}$ & $26.8 \mathrm{a}$ & $27.7 b c$ & $28.5 \mathrm{a}$ & $28.1 \mathrm{a}$ & $19.0 \mathrm{c}$ & $18.2 \mathrm{e}$ & $18.6 \mathrm{c}$ & $19.9 \mathrm{c}$ & $19.2 \mathrm{~d}$ & $19.6 \mathrm{c}$ \\
\hline & $\mathbf{T}$ & $0.38 b$ & $0.40 \mathrm{a}$ & & $26.3 b$ & $26.8 \mathrm{a}$ & & $27.4 \mathrm{~b}$ & $28.0 \mathrm{a}$ & & $19.5 \mathrm{a}$ & $18.9 \mathrm{~b}$ & & $20.5 \mathrm{a}$ & $19.9 \mathrm{~b}$ & \\
\hline & Control & & 0.26 & & & 26.8 & & & 27.9 & & & 18.1 & & & 19.4 & \\
\hline & omp. of means & & S.Em. \pm & & & S.Em. \pm & & & S.Em. \pm & & & S.Em. \pm & & & S.Em. \pm & \\
\hline & I & & 0.01 & & & 0.17 & & & 0.18 & & & 0.33 & & & 0.33 & \\
\hline & $\mathbf{F}$ & & 0.01 & & & 0.13 & & & 0.14 & & & 0.08 & & & 0.07 & \\
\hline & $\mathbf{T}$ & & 0.01 & & & 0.10 & & & 0.11 & & & 0.06 & & & 0.06 & \\
\hline & $\mathbf{I} \times \mathbf{F}$ & & 0.01 & & & 0.22 & & & 0.24 & & & 0.13 & & & 0.13 & \\
\hline & $\mathbf{I} \times \mathbf{T}$ & & 0.01 & & & 0.18 & & & 0.19 & & & 0.11 & & & 0.10 & \\
\hline & $\mathbf{F} \times \mathbf{T}$ & & 0.01 & & & 0.18 & & & 0.19 & & & 0.11 & & & 0.10 & \\
\hline & $\mathbf{I} \times \mathbf{F} \times \mathbf{T}$ & & 0.02 & & & 0.31 & & & 0.34 & & & 0.19 & & & 0.18 & \\
\hline & ont. v/s treats. & & 0.02 & & & 0.33 & & & 0.34 & & & 0.35 & & & 0.36 & \\
\hline
\end{tabular}

\begin{tabular}{|c|c|c|c|}
\hline Residual effect of irrigation levels (I) : & Residual effect of fertilizer levels $(\mathbf{F})$ : & Residual effect du & application (T): \\
\hline $\mathbf{I}_{1}$ - Deficit irrigation (growth stages) & $\mathrm{F}_{1}-200: 75: 75 \mathrm{~kg} \mathrm{~N}: \mathrm{P}_{2} \mathrm{O}_{5}: \mathrm{K}_{2} \mathrm{O} \mathrm{ha}^{-1}(2.67: 1: 1)$ & $\mathrm{T}_{1}-25 \%$ of $\mathrm{N}, \mathrm{K}_{2} \mathrm{O}$ and $50 \%$ of $\mathrm{P}_{2} \mathrm{O}_{5}$ as basal, & $\mathrm{T}_{2}-33.3 \%$ each of $\mathrm{N}, \mathrm{P}_{2} \mathrm{O}_{5}$ and $\mathrm{K}_{2} \mathrm{O}$ as basal, \\
\hline$I_{2}-I_{1}+$ hydrogel $\left(2.5 \mathrm{~kg} \mathrm{ha}^{-1}\right)$ & $\mathrm{F}_{2}-225: 100: 100 \mathrm{~kg} \mathrm{~N}: \mathrm{P}_{2} \mathrm{O}_{5}: \mathrm{K}_{2} \mathrm{O} \mathrm{ha}^{-1}(2.25: 1: 1)$ & $50 \%$ of $\mathrm{N}, \mathrm{P}_{2} \mathrm{O}_{5}$ and $\mathrm{K}_{2} \mathrm{O}$ at $30 \mathrm{DAS}$ and & $33.3 \%$ each of $\mathrm{N}_{2} \mathrm{P}_{2} \mathrm{O}_{5}$ and $\mathrm{K}_{2} \mathrm{O}$ at $30 \mathrm{DAS}$ and \\
\hline $\begin{array}{c}I_{3} \text { - Alternatively alternate furrow irrigation (50 \% depletion of soil } \\
\text { moisture) }\end{array}$ & $\begin{array}{l}\mathrm{F}_{3}-250: 100: 125 \mathrm{~kg} \mathrm{~N}: \mathrm{P}_{2} \mathrm{O}_{5}: \mathrm{K}_{2} \mathrm{O} \mathrm{ha}^{-1} \\
(2.5: 1: 1.25)\end{array}$ & $25 \%$ of $\mathrm{N}$ and $\mathrm{K}_{2} \mathrm{O}$ at 50 DAS & $33.3 \%$ each of $\mathrm{N}, \mathrm{P}_{2} \mathrm{O}_{5}$ and $\mathrm{K}_{2} \mathrm{O}$ at 50 DAS \\
\hline
\end{tabular}




\section{References}

Ali, M., Dahan, R., Mishra, J. P., Saxena, N. P., 2000, Towards the more efficient use of water and nutrients in food legume cropping. In: Knight, R. (eds) Linking research and marketing opportunities for Pulses in the 21st century. Current Plant Science and Biotechnology in Agriculture, 34. Springer, Dordrecht. pp. 355-368.

Bandyopadhyay, P. K., Singh, K. C., Mondal, K., Nath, P., Ghosh, P. K., Kumar, N., Basu, P. S. and Singh, S. S., 2016, Effects of stubble length of Rice in mitigating soil moisture stress and on yield of Lentil (Lens culinaris Medik) in Rice - Lentil relay crop. Agricultural Water Management, 173: 91-102.

Bhattacharyya, R., Pandey, S. C., Chandra, S., Kundu, S., Supradip Saha, Mina, B. L., Srivastva, A. K. and Gupta, H. S., 2010, Fertilization effects on yield sustainability and soil properties under irrigated Wheat (Triticum aestivum L.) - Soybean (Glycine max L.) rotation of an Indian Himalayan upper valley. Nutrient Cycling in Agroecosystem, 86(2): 255-268.

De, J. K., 2003, Residual effect of Black gram in Maize - Black gram cropping sequence in Ultic Hapludalf of Assam.
Indian Journal of Agricultural Sciences, 73(2): 75-78.

Donald, C. M., 1962, In search of yield. Journal of Australian Institute of Agriculture Science, 28: 171-178.

Gan, Y., Hamel, C., Kutcher, H. R. and Poppy, L., 2016, Lentil enhances agro ecosystem productivity with increased residual soil water and nitrogen. Renewable Agriculture and Food Systems, 32(4): 319-330.

Gomez, K. A. and Gomez, A. A., 1984, Statistical Procedure for Agricultural Research. John Wiley and Sons, New York, pp. 130-271.

Hazra, K. K. and Bohra, A., 2021, Increasing Relevance of Pulse crops to sustainable intensification of Indian Agriculture. National Academy Science Letters, 44: 1-3.

Kumar, N., Yadav, A., Singh, S. and Yadav, S. L., 2018, Growing pulses in Rice fallow: ensuring nutritional security in India. Conservation Agriculture for Advancing Food Security in Changing Climate, 1: pp. 107-122.

Shrestha, B. B. and Roy, P., 2016, Utilisation of residual soil moisture through relay cropping. Working Paper, pp. 1-51.

Singh, K. K., Ali, M. and Venkatesh, M. S., 2009, Pulses in Cropping Systems. Technical Bulletin, IIPR, Kanpur. pp. $1-47$.

\section{How to cite this article:}

Sangh Ravikiran and Angadi, S. S. 2021. Irrigation and Fertilizers Management Practices in Maize (Zea mays L.) and their Residual Effect on Harvest Index and Soil Moisture Studies of Soybean (Glycine max L.). Int.J.Curr.Microbiol.App.Sci. 10(02): 3141-3145. doi: https://doi.org/10.20546/ijcmas.2021.1002.344 